\title{
Dawn on the mice
}

By directly delivering light deep into the brain, scientists can now study the basis of neurological therapy and animal behavior.

The mammalian brain is a secluded and enigmatic organ. Despite the progress in molecular and cellular biology, we still know very little about the brain circuitry and how it controls behavior. One of the challenges of brain studies is accessibility. The brain is protected by the opaque skull, which makes biochemical and genetic manipulations difficult.

One way to access deep inside the brain is to insert thin electrodes through the skull. Deep brain stimulation is one of these minimally invasive methods and is used to treat Parkinson's disease and depression. Deep brain stimulation electrically stimulates the subthalamic nucleus, a complex structure with neurons, glia, and efferent and afferent fibers. "[Deep brain stimulation] is a fundamentally mysterious intervention. There is not much knowledge, but plenty of hypotheses, in terms of the circuit element that's been recruited,' explains to Karl Deisseroth, a psychiatrist and neuroscientist at Stanford University.

Deisseroth's group has pioneered a versatile approach called optogenetics, which uses light-gated microbial ion channels to control neuron membrane potential. Channelrhodopsin-2 (ChR2) is a light-

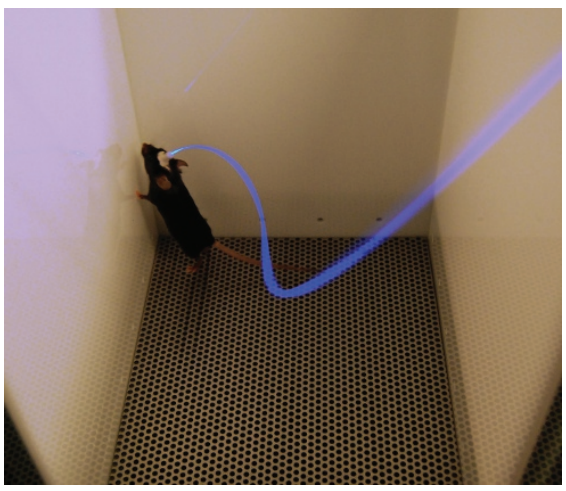

Mouse with optrode inserted for targeted nucleus accumbens stimulation. Blue light is delivered to the optrode via an optical fiber linking the laser diode and the freely moving mouse. Image courtesy of K. Deisseroth. activated cation channel that can excite a neuron. Halorhodopsin (NpHR), in contrast, is a chloride pump that can inhibit a neuron. As with electrical stimulation, photostimulation offers precise temporal control.

Deisseroth is interested in using optogenetics to help understand how deep brain stimulation works and to improve the procedure. Viviana Gradinaru, a graduate student in his laboratory, has designed a hybrid instrument called an 'optrode', which consists of fiber optics for photostimulation and an electrode for activity recording. By inserting an optrode precisely into a specific brain region, she can simultaneously photostimulate the area and measure electrical activity there.

To understand how deep brain stimulation works, Deisseroth and colleagues expressed ChR2 or NpHR in different subthalamic nucleus cell populations in a Parkinson's disease model (Gradinaru et al., 2009). They stimulated the subthalamic nucleus with an optrode to see whether any cell type-specific manipulation reverses the behavioral defect of the model. After systematically testing excitatory neurons and astroglia without seeing a therapeutic effect, they finally found that the afferent fibers originated from the cortex as an important target of deep brain stimulation.

Meanwhile, another student in Deisseroth's laboratory, Raag Airan, was developing a radical approach to broaden the reach of optogenetics. In addition to electrical signals, biochemical signals are also important in the brain. "This axis [of biochemical signals] is not directly accessible to microbial opsins," says Deisseroth. Airan engineered genetically encoded optical tools called 'optoXRs' consisting of extracellular and transmembrane domains of bovine rhodopsin and intracellular loops of another G protein-coupled receptor to control a distinct intracellular signaling pathway (Airan et al., 2009). They used $\beta_{2}$-adrenergic receptor loops (opto$\beta_{2} \mathrm{AR}$ ) to elevate cAMP and loops from the $\alpha_{1}$-adrenergic receptor (opto- $\alpha_{1} A R$ ) to mobilize calcium. With this repertoire of photoactivated $\mathrm{G}$ protein-coupled receptor chimeras, signaling pathways can be controlled with fine temporal precision.

To test the optoXRs in vivo, Deisseroth and colleagues expressed them in brain neurons and used the optrode to stimulate and record activity. They found that spike frequency in the nucleus accumbens, the reward center in the brain, decreases and increases upon photoactivation of opto- $\beta_{2} \mathrm{AR}$ and opto- $\alpha_{1} A R$, respectively. They thus set up a behavioral paradigm to test whether high nucleus accumbens activity encodes reward memory. They allowed a mouse with opto$\alpha_{1} A R$ expression in the nucleus accumbens to freely roam for one day. On the second day, whenever the mouse entered the right side of the cage, they sent light via an optrode to the nucleus accumbens to induce reward memory. On the third day, they sent no light, but the mouse now preferred to stay on the right side of the cage in expectation of receiving the reward stimulation. Therefore, optoXRs can control neuronal activity and mouse behavior through biochemical signals.

These reports demonstrate the versatility of optogenetics. A major consideration is the availability of the light-absorbing retinoids needed to form the rhodopsin chromophore. "It is possible that retinoid may be used throughout mammalian tissue for trophic and signaling roles; most tissues will likely have sufficient retinoids," says Deissseroth. In addition to the nucleus accumbens, other brain areas, such as the amygdala, and behaviors, such as anxiety, can be studied by optogenetics. Finally, with additional genetic engineering, optoXRs for other signaling pathways can be made. It has begun to dawn on the mice, and on us, too, that the growing optogenetics toolbox will be invaluable in revealing the mysteries of the brain.

\section{Wayne Peng}

\section{RESEARCH PAPERS}

Airan, R.D. et al. Temporally precise in vivo control of intracellular signalling. Nature advance online publication (18 March 2009).

Gradinaru, V. et al. Optical deconstruction of Parkinsonian neural circuitry. Science advance online publication (19 March 2009). 